\title{
Comparison between osseodensification burs and osteotome technique for closed sinus lift in partially edentulous maxilla (clinical and radiological study)
}

\author{
By \\ Dr. Ali Nahi Hamdi ${ }^{* 1}$; Dr. Shehab Ahmed Hemd ${ }^{* 2}$ \\ * 1 B.D.S M.Sc. Candidate/ Oral surgery / college of dentistry / Hawler medical university; \\ ${ }^{*}{ }^{2}$ Assist prof. / Oral surgery / college of dentistry / Hawler medical university
}

D.O.I - 10.51201/JUSST/21/05164

http://doi.org/10.51201/JUSST/21/05164

\section{$\underline{\text { Abstract }}$}

Dental implants are considered the first choice to replace lost or non-restorable teeth. However, the posterior maxilla remains a challenge in its management because of the quality of bone in the posterior maxilla. Osseodensification (OD) concept has been proposed in the literature to improve primary implant stability, which is an important aspect of osseointegration. Densah bur is novel drills specially designed to enhance a bone density by Osseodensification, which in turn increases primary stability.This present study was conducted to assess crestal sinus floor elevation by osteotome in comparison to densah bur in the posterior atrophic maxilla. This was a randomized controlled clinical trial conducted on 20 patients to evaluate available crestal bone height loss, implant stability after implant placement in healed posterior maxillary alveolar ridge, whole bone height, Schneiderian membrane trauma, and post-operative complication.

All crestal sinus floor elevations were performed for 20 patients with at least $5 \mathrm{~mm}$ residual bone height. 10 randomly selected patients received osteotome sinus elevation (group 1), and 10 received osseodensification sinus elevation (group 2), were recalled 2 days after the operation, then after four-month until the prosthetic 
phase. The clinical assessment involved the assessment of postoperative sequelae including edema, pain and postoperative healing. The evaluation involved clinically and radiographically. Periapical radiographs and orthopantogram took for every patient immediately at the time of fixture insertion and after 4 months postoperatively to evaluate crestal bone height loss. While implant stability was obtained by using, ratchet and periotest device immediately after implant insertion and 4 months postoperatively at the time of implant exposure.

keywords: osseodensification burs ; osteotome technique ; closed sinus lift ; partially edentulous maxilla

\section{Introduction}

The use of dental implants is considered a safe and reliable procedure for replacing missing teeth ${ }^{(1)}$. Dental implants placement is a challenging procedure in the presence of unfavourable local condition of the alveolar ridge ${ }^{(2)}$ In the posterior sextants of the maxilla, tooth loss is generally associated with alveolar bone loss and sinus pneumatisation ${ }^{(3)}$, due to loss of mechanical function after tooth extraction or tooth $\operatorname{loss}^{(4)}$. In addition, poor bone quality, volume and difficult access may have a negative influence on the survival rate of implants ${ }^{(5)}$ However, it is not possible to place dental implants with an adequate length in some clinical situations, primarily due to the lack of sufficient bone ${ }^{(6)}$.

Thus, an augmentation procedure is often indicated in this area. Maxillary sinus lift is one of the most common surgical techniques ${ }^{(7)}$ it has been considered a safe treatment modality with a low complication rate ${ }^{(8)}$. Several systematic reviews of the literature showed high overall implant survival rates well beyond $90 \%$ for sinus floor evaluation $^{(9)}$. 
However, the edentulous ridge in the posterior maxilla often presents limited bone volume due to both lack of alveolar bone after ridge remodeling and to maxillary sinus pneumatization ${ }^{(10)}$ Also, it can be observed that the grafting materials studied can be used safely for transcrestal lifting the maxillary sinus ${ }^{(11) .}$

And so the aim of sinus lift procedure is to compensate this bone loss by creating bone volume in the maxillary sinus and thus enabling installation of dental implants. ${ }^{(12) .}$ The main risk factor for implant failure is the primary stability of the implant ${ }^{(13) .}$ Bone density and especially cortical thickness are important factors in achieving adequate primary stability ${ }^{(14) .}$

Secondary stability is defined as stability comes after osseointegration ${ }^{(15) .}$, is the biologic stability provided through bone regeneration and remodelling ${ }^{(16) .}$ Both parameters are interrelated positively, which would ensure the likelihood of implant success and osseointegration ${ }^{(17) .}$ Osseointegration is the result of initial mechanical (primary) stability complemented by biological stability; the sum of these two parameters will give the value of the final stability ${ }^{(18) .}$

\section{Patients and method}

The study was planned to be performed in Khanzad dental specialist center , Erbil city , February 2020 to June 2021. The data was to be collect by direct interview and the time of the interview approximately was between $10-15$ minutes. The questions to be directed to patients were willing to participate.

Do dental implants inserted with the use of osseodensification drilling technique compared to a conventional osteotome technique show improved performance in terms of stability.Do dental implants inserted with the use of an osseodensification drilling technique compared to conventional osteotome technique present more interfacial bone tissue around dental implants. 
In patients undergoing dental implant, does the use of an osseodensification drilling technique in one group compared to a conventional osteotome technique in the second group show a higher survival rate and less marginal bone loss.

A randomly systematic sampling technique was to be used by a selection of 10 delayed-loaded dental implant(s) with osseodensification and 10 delayed-loaded dental implant(s) with conventional osteotome technique placed in conjugation with crestal maxillary sinus floor elevation, Patients were classified into two equal groups: Group (A):Ten patients were received delayed-loaded implant(s) with crestal maxillary sinus floor elevation using osteotomes and drills and Group (B): Ten patients were received delayed-loaded implant(s) with crestal maxillary sinus floor elevation using osseodensification technique (OD) by densah burs.

\section{Methods:}

All surgical procedures were performed under strict aseptic conditions.

Patient was instructed to rinse his/her mouth with $0.12 \%$ chlorhexidine gluconate 4 for 1 minute before starting surgical procedures. A midcrestal incision is made using No. 15 blade and Flap reflection (mucoperiosteal flap: buccal and palatal).

The implant site is marked with a $2.0 \mathrm{~mm}$ pilot drill and then prepared with a drill to a depth of $1 \mathrm{~mm}$ (according to OPG) from the sinus floor to prevent the tip of the drill from rupturing the Schneiderian membrane.For group A (closed sinus lifting by Osteotome), A concave osteotomes set of varying dimensions can be sequentially used to widen the osteotomy site by surgical mallet. A $2.2 \mathrm{~mm}$ osteotome is inserted into the osteotomy and advanced with light malleting to a depth of $1 \mathrm{~mm}$ from the sinus floor then followed by $2.7 \mathrm{~mm}$ osteotome to the same depth, A $3.2 \mathrm{~mm}$ osteotome is tapped gently to fracture up the sinus floor.Then a $3.7 \mathrm{~mm}$ osteotome is tapped gently to elevate the sinus floor to 
desired depth of the implant in the maxillary sinus, after sinus floor fracture, alloplastic graft material was prepared by mixing with saline then applied to osteotomy site to working depth by $4.2 \mathrm{~mm}$ osteotome then 4.7 osteotome, then sealed sterile implant package was opened and the implant was guided into its position with light stable finger pressure. The coupling wrench with ratchet was used to complete installation of implant $0.5 \mathrm{~mm}$ below alveolar crestal bone.

Measurement of implant stability was immediately performed during implant placement by measuring implant insertion torque (IT), using a customized manual torque ratchet (multi torque setting wrench can be adjusted to 15, 20, 25, 30, 35, 40, 45 and $50 \mathrm{Ncm}$ ), and after implant placement with aid of resonance frequency analysis (RFA)device. The analysis of RFA was made by using an Osstell beacon• apparatus with a commercially available transducer(smart peg type 49, article N0. DSP01) adapted to the implant by hand tightening on to implant fixture with a torque of 5-10 Ncm. The transducer was maintained perpendicular to the implant and was hand screwed into the implant body as recommended by the manufacturer. The Implant Stability Quotient ISQ values were measured both parallel and perpendicular to alveolar ridge of the jaw. The ISQ value for each implant was the mean of ISQ values taken from all directions. ISQ values were measured again at re-entry after 4 months for each implant. After assessment of primary stability, the transducer was removed and the surgical covering screw was applied over the implant fixture. Then, the mucoperiosteal flap was repositioned and primary closure was achieved using a combination of mattress and interrupted sutures by $4 / 0$ silk suture material.

An immediate postoperative periapical x-ray was taken to verify the final position of the implant and assist the crestal bone level For group B (closed sinus lifting by densah bur), Change the drill motor to reverse- densifying Mode (counterclockwise 
drill speed 800 rpm with copious irrigation) with torque 40 NCM. Begin with the densah bur (2.5mm) until $1 \mathrm{~mm}$ short of the sinus floor.

Use the next wider Densah Bur (3.0) and advance it into the previously created osteotomy with modulating pressure and a pumping motion. When feeling the haptic feedback of the bur reaching the dense sinus floor, modulate pressure with a gentle pumping motion to advance past the sinus floor. Maximum advancement past the sinus floor at this stage must not exceed $2.0 \mathrm{~mm}$.

As the next wider densah burs $(3.5 \mathrm{~mm})$ advance in the osteotomy, the bone pushed toward the apical end and begin to gently lift the membrane and autograft compacted bone to achieve additional vertical depth and membrane lift of $2.0 \mathrm{~mm}$ and reach final desired width for implant placement. Then alloplastic bone substitute was placed into the final width osteotomy using the final diameter densah bur (4.5 mm) in densifying mode (Counterclockwise drill speed 200-600 rpm with slow irrigation) with gentle pumping motion facilitating the graft material compaction and further lifting of the sinus. Then implant was inserted into the final desired depth. Then implant primary stability was assessed and after that implant covered with cover screw and flap repositioned and sutured as done as in group (A). An immediate postoperative x-ray was taken to verify the final position of the implant, the condition of the sinus and the level of crestal alveolar bone.

For both groups, the integrity of the sinus membrane was examined using the Valsava maneuver by blocking the patient's nostrils and asking the patient to blow through his or her nose.After 7 days' appointment to record post-operative complication like pain and swelling and for suture removal. Then after four months the gingival form will be placed on implant and the secondary stability recorded by osstell.Suturing the flab around gingival form. 
Postoperative management: Patients were instructed to maintain good oral hygiene, avoid solid textured food or blowing through their nose and to use $0.2 \%$ chlorhexidine gluconate solution as a mouth rinse for 2 weeks. Postoperative medication consisted of continuing the course of antibiotic (1 g amoxicillin/ clavulanic acid twice daily for 5 days after surgery). Ibuprofen $400 \mathrm{mg}$ was prescribed as analgesic 3 times per day. Otrivin6 nasal drops were used 3 times a day as nasal decongestant for the first three days only to avoid rebound effect. Sutures were removed 1 week after surgery.

The Prosthetic Phase:Second stage surgery was performed 4 months from surgical installation of implant fixture by making a crestal incision to expose the implant platform then placing of healing cap for soft tissue healing around implant. After 2 weeks an open tray impression technique was taken to fabricate cement retained porcelain fused metal (PFM) restorations. Final prosthesis was cemented after checking marginal adaptation and occlusal contacts.

All patients were evaluated clinically and radiographically during a follow up period of 4 months after operation where the following criteria were evaluated, so pain assessment and Implant stability, and crestal Bone Height assessment.

Clinical Evaluation:Pain was assessed immediately after surgery and one week after surgery; using The 0-to-10 Numerical Rating Scale (NRS).170 Patient was asked to select the number that best represents his/her current pain intensity or discomfort on a 0 -to-10 scale, where $0=$ No pain or total satisfaction and $10=$ Severe pain or total discontent. The number that the respondent selects is that respondent's NRS score. Pain intensity can be classified into mild, moderate, and severe levels based on the Numerical Rating Score NRS. Mild pain included scores from 1-3 on NRS; with noticeable pain with little effect on day-to-day functioning. Moderate pain included scores from 4-6; pain interfering with sleep and mood. 
Severe pain included scores from 7-10; with pain interfering with wide range of activities.

Implant stability in all patients were assessed by recording insertion torque (IT) and by the ISQ (implant stability quotient) levels measured by Osstell ISQ device (Fig.4). Readings were taken immediately after fixture insertion (T0) and at second stage surgery (4 months after implant placement) (T4). Resonance frequency analysis (RFA) values expressed as (ISQ) was recorded by a transducer attached to the implant by a screw and a frequency response analyzer (Osstell ISQ Device) with the average of 2 measurements performed with the probe in 2 perpendicular directions. so ISQ scale is from 0 to 100 and is a measure of the stability of an implant. The ISQ scale has no linear correlation to micromobility. Scales > 70 ISQ means high stability, scales between 60-69 ISQ means medium stability and scales $<60$ ISQ means low stability.

Statistical analysis: Data management and statistical analysis were performed using the Statistical Package for Social Sciences (SPSS) version. 22.

\section{Results :}

Twenty patients who met inclusion criteria and needed fixed replacement of edentulous posterior maxillary area with delayed-loaded dental implant(s) placed in conjugation with crestal maxillary sinus floor elevation were included in the study. Twenty patients (13 females and 7 males) were divided into two groups:

Group A: Ten patients received delayed-loaded implant(s) with crestal maxillary sinus floor elevation using osteotomes and drills. Group B: Ten patients received delayed-loaded implant(s) with crestal maxillary sinus floor elevation using densah burs.Gender distribution among groups was 6 females and 4 males in group A, 7 females and 3 males in group B (Table 1).Comparing both groups, no statistical 


\section{\begin{tabular}{l|l} 
Group A & Group B \\
\hline
\end{tabular}}

significant difference were recorded regarding to patient age ( $\mathrm{p}$ value $=0.48$ ) and statistically with significant different for gender $\mathrm{P}=0.05$.

Table (1): Showing descriptive statistics, Fisher exact and independent t-test for the age and gender of the two groups

\begin{tabular}{|c|c|c|c|c|c|c|}
\hline \multicolumn{2}{|l|}{$\begin{array}{l}\text { Group } \\
\text { Variables }\end{array}$} & \multicolumn{2}{|c|}{$\begin{array}{l}\text { Group A } \\
(n=10)\end{array}$} & \multicolumn{2}{|c|}{$\begin{array}{l}\text { Group B } \\
(\mathrm{n}=10)\end{array}$} & $P$ value \\
\hline \multirow[t]{3}{*}{ Age (yrs.) } & Mean \pm SD & \multicolumn{2}{|c|}{$40.9 \pm 8.5$} & \multicolumn{2}{|c|}{$43.9 \pm 9.6$} & $0.49^{\#}$ \\
\hline & \multirow[t]{2}{*}{ Range } & \multicolumn{2}{|c|}{$24-53$} & \multicolumn{2}{|c|}{$29-56$} & \\
\hline & & No. & $\%$ & No. & $\%$ & \\
\hline \multirow[t]{2}{*}{ Gender } & Female & 6 & 60 & 7 & 70 & \multirow[t]{2}{*}{$0.33^{*}$} \\
\hline & Male & 4 & 40 & 3 & 30 & \\
\hline
\end{tabular}

\# Independent t-test * Fisher exact test

Comparing both groups, no statistical significant difference were recorded regarding to patient age and gender ( $\mathrm{p}$ value $=0.49$ and 0.33 ).

Pain Assessment [Numerical Rating Score (NRS):

Immediately (T0), the median and range of the NRS scores (Table 2) was 4.5(3-7) for Group A and 2(2-4) for Group B with statistically significant difference between both groups $(\mathrm{p}=0.000)$. After 1 week, the median and range of the NRS scores was 3(2-4) for Group A and 1(0-2) for Group B with statistically significant difference between both groups $(\mathrm{p}<0.05)$. Comparing median pain score over time in each single group was statistically significant. $(\mathrm{p}<0.05)$.

Table (2): Showing Median and range of NRS score at different time points. Mann Whitney test and Friedman Test were used. 


\begin{tabular}{|l|l|l|l|l|l|l|l|l|l|}
\hline NRS. & Mean & STD & $\begin{array}{l}\text { Minimu } \\
\mathrm{m}\end{array}$ & Maximum & Mean & STD & Minimum & $\begin{array}{l}\text { Maxi } \\
\text { mum }\end{array}$ & P value1 \\
\hline $\begin{array}{l}\text { Immediate } \\
(\mathrm{T} 0)\end{array}$ & 4.9 & 1.37 & 3 & 7 & 2.9 & $\begin{array}{l}0.7 \\
0\end{array}$ & 2 & 4 & 0.00 \\
\hline 1Week & 2.5 & 0.78 & 2 & 4 & 0.9 & 0.7 & 0 & 2 & 0.00 \\
\hline P value2 & 0.00 & & & & 3 & & & \\
\hline
\end{tabular}

Implant stability quotient (ISQ) values were measured at T0 and T4 using osstell ISQ device. (Table 3)

Implant stability at T0: For group A; the ISQ values at T0 ranged from 52 to 75 $\mathrm{N} / \mathrm{cm}$, with mean value of $60.3 \pm 7.2$. For group B; the ISQ values at T0 ranged from 51 to $82 \mathrm{~N} / \mathrm{cm}$ with a mean value of $66 \pm 8.8 \mathrm{~N} / \mathrm{cm}$. There was no statistically significant difference between the two groups $(\mathrm{P}=.0 .19)$.

Implant Stability at T4: Implant stability was measured at the time of abutment connection (3 months after implant placement; T4). For group A; the ISQ values at T4 ranged from 60 to 76 with a mean of $66.9 \pm 4.5$.

For group B; the ISQ values at T4 ranged from 60 to 78 with a mean of $71.8 \pm 5.5$. There was statistically significant difference between the two groups at $\mathrm{T} 4(\mathrm{P}=$ $0.01)$.

Table 3: Showing no statistical difference regarding implant stability quotient (ISQ) values between two groups at T0 and there was a statically significance difference at $\mathrm{T} 4$

\begin{tabular}{|c|c|c|c|c|c|c|c|}
\hline \multirow{3}{*}{$\begin{array}{l}\text { Implant } \\
\text { Stability } \\
\text { Quotient }\end{array}$} & \multicolumn{6}{|c|}{ Groups } & \\
\hline & \multicolumn{3}{|c|}{$\begin{array}{l}\text { Group A } \\
\text { (Osteotome) }\end{array}$} & \multicolumn{3}{|c|}{$\begin{array}{l}\text { Group B } \\
\text { (Densah burs) }\end{array}$} & \\
\hline & Mean & SD & (Range ) & Mean & SD & (Range ) & $\mathrm{P}$ value1 \\
\hline
\end{tabular}




\begin{tabular}{|l|l|l|l|l|l|l|l|}
\hline $\begin{array}{l}\text { Values } \\
\text { (ISQ) }\end{array}$ & & & & & & & \\
\hline T0 & 60.3 & 7.2 & $52-75$ & 66 & 8.8 & $51-82$ & 0.19 \\
\hline T4 & 66.9 & 4.5 & $60-73$ & 71.8 & 5.5 & $60-78$ & 0.01 \\
\hline P value2 & 0.03 & & 0.13 & & \\
\hline
\end{tabular}

For group A; the ratchet values ranged from 25 to $45 \mathrm{~N} / \mathrm{cm}$, with mean value of $33 \pm 7.5$. For group $B$; the ratchet values ranged from 20 to $45 \mathrm{~N} / \mathrm{cm}$ with a mean value of $33 \pm 6.1 \mathrm{~N} / \mathrm{cm}$. There was statistically not significant difference between the two groups $(\mathrm{P}=0.75)$ (Table 4$)$.

Table 4: showing statically not significance deference considered the insertion torque between the two groups

\begin{tabular}{|c|c|c|c|c|c|c|c|}
\hline \multirow{3}{*}{$\begin{array}{l}\text { ratchet } \\
\text { torque }\end{array}$} & & & & & & & \\
\hline & \multicolumn{3}{|c|}{ (Osteotome) } & \multicolumn{3}{|c|}{ (Densah burs) } & \\
\hline & Mean & SD & (Range ) & Mean & SD & (Range ) & P value \\
\hline $\begin{array}{l}\text { Ratchet } \\
\text { reading }\end{array}$ & 33 & 7.5 & $25-45$ & 34 & 6.1 & $20-45$ & 0.75 \\
\hline
\end{tabular}

The Amount of crestal bone Loss in both groups presented in Table (5) The mean marginal bone loss of group A was $0.75 \pm 0.20 \mathrm{~mm}$ compared to $0.71 \pm 0.11 \mathrm{~mm}$ in group B. This was statistically not significant; $\mathrm{p}=0.45$.

Table 5: Showing statistically not significant difference regarding crestal bone height within each group 


\begin{tabular}{|c|c|c|c|c|c|c|c|c|c|c|}
\hline \multirow{2}{*}{$\begin{array}{l}\text { Amount } \\
\text { of bone }\end{array}$} & \multicolumn{4}{|c|}{ Group A (osteotomes ) } & \multicolumn{5}{|c|}{ Group B (densah bur ) } & \multirow{2}{*}{$\begin{array}{l}\mathrm{P} \\
\text { value }\end{array}$} \\
\hline & Mean & SD & MAX. & MIN. & Mean & SL & & MAX. & MIN. & \\
\hline \multicolumn{11}{|l|}{ loss } \\
\hline \multicolumn{5}{|c|}{ Group A } & \multicolumn{6}{|c|}{ Group B } \\
\hline NRS. & Mean & STD & $\begin{array}{l}\text { Minimu } \\
\mathrm{m}\end{array}$ & Maximum & Mean & STD & \multicolumn{2}{|c|}{ Minimum } & $\begin{array}{l}\text { Maxi } \\
\text { mum }\end{array}$ & P value1 \\
\hline $\begin{array}{l}\text { Immediate } \\
\text { (T0) }\end{array}$ & 4.9 & 1.37 & 3 & 7 & 2.9 & $\begin{array}{l}0.7 \\
0\end{array}$ & \multicolumn{2}{|c|}{2} & 4 & 0.00 \\
\hline 1Week & 2.5 & 0.78 & 2 & 4 & 0.9 & $\begin{array}{l}0.7 \\
3\end{array}$ & \multicolumn{2}{|c|}{0} & 2 & 0.00 \\
\hline P value2 & \multicolumn{4}{|l|}{0.00} & \multicolumn{5}{|l|}{0.00} & \\
\hline & 0.75 & 0.20 & 1.1 & 0.05 & 0.71 & 0. & & 0.95 & 0.50 & 0.45 \\
\hline
\end{tabular}

\section{Discussion}

Posterior maxilla is always a challenging site for dental implant placement due to number of anatomic challenges. Atrophy of the edentulous ridge in buccolingual and apico-occlusal direction and pneumatization of the maxillary sinus often reduce the volume of bone available for implant placement. Residual bone in the posterior maxilla is usually type IV in quality. Such poor-quality bone has been cited as a significant contributing factor to greater implant failure in a number of studies

(19\&20)

Several techniques have been proposed in literature concerned with increasing local bone volume, thus allowing placement of implants in posterior maxilla in patients with insufficient bone height. Many techniques have been advocated for this purpose like lateral sinus lifting, guided bone regeneration (GBR) and onlay block graft. Most of these procedures include long waiting periods of 6-12 months, 
increased morbidity, and an additional surgical site with increased cost to the patient $^{(21 \& 22)}$

Maxillary sinus floor elevation through crestal approach is a fairly common procedure for implant rehabilitation of posterior maxilla with successful outcome in means of grafting technique and long-term implant survival rate (ISR) ${ }^{(23)}$

The hypothesis of this study was that the use of osseodensification burs for crestal sinus floor elevation could yield better results in terms of implant stability and patient acceptance when compared to the traditional osteotome techniques.

In our study All implants in both groups had ISQ values ranging from 52 to 71 for group A and from 51 to 82 for group B indicating fair primary stability which is very important for success of dental implant ${ }^{(24 \& 25)}$. Turkyilmaz et al. found a positive strong correlation between bone density (calculated with computed tomography) and ISQ values, he reported that high ISQ values recorded (70.5 46$)$ resulted from the higher quality of bone in the anterior mandible, the surgical technique with no pre-tapping, and the roughened-surface implants used ${ }^{(26)}$. The significant difference of implant stability observed within each group when comparing implant stability after 4 months to initial stability. Then ISQ values showed slight increase for both groups when measured after 4 months of implant insertion. This change in stability matched the pattern of stability with implants placed in routine procedure without sinus lifting procedure. The variation in ISQ values is reflective of the biologic changes at the bone implant interface. This finding is similar to ${ }^{(27,28)}$

The results from our study concluded that there is statistically significant difference between the control and test groups; $\mathrm{p}=0.02$. The mean primary implant stability of group A (osteotome) was $6.3 \pm 7.2$ compared to $66.0 \pm 8.8$ in group B 
(densah bure). After 4 months, the mean secondary implant stability of group A was $66.9 \pm 4.5$ compared to $71.8 \pm 5.5$ in group B. When it comes to implant stability many publications offer differing views. for example, claimed that bone compaction technique through the OD drilling increased the insertion torque, boneto-implant contact, and accordingly resulted in greater primary stability compared to conventional drilling. They proposed that the osseodensification technique conserves bone by compaction of cancellous bone due to viscoelastic and plastic deformation, and compaction autografting of bone particles along the length and at the apex of the osteotomy. Which meet the results from our study that there is statistically not significant difference between the control and test groups; $\mathrm{p}=0.75$. The mean insertion torque for group A (osteotome) was $33 \pm 7.5$ compared to $34 \pm 6.1$ in group B (densah bure). Regarding crestal bone height, there was no statistical difference between both groups at different times of follow up. Two periapical images were obtained for each implant immediately and 4 months postoperatively to assess marginal bone loss. The mean marginal bone loss of group A (osteotome) was $0.75 \pm 0.20 \mathrm{~mm}$ compared to $0.71 \pm 0.11 \mathrm{~mm}$ in group B (densah bure). This was statistically not significant; $\mathrm{p}=0.45$.

As patient discomfort, pain and benign paroxysmal vertigo has been reported after osteotome mediated sinus floor elevation. Patients were asked to give their perception on the surgical procedure. Patient discomfort and pain was evaluated immediately after surgery and after one week of the surgery. NRS scores were used for this purpose; in which (0) indicated total satisfaction and no pain and (10) indicated total discontent ${ }^{(29)}$.

Comparing NRS scores for both groups immediately; patients in group (A) showed moderate pain and discomfort scores, while group (B) patients showed mild pain. Poor patients experience in group (A) may be related to percussive forces exerted 
by malleting with osteotome, although the osteotome usage in our study was limited to is the step of fracturing remaining thin sinus floor.A lot of authors have reported patient discomfort and Benign Positional Paroxysmal Vertigo (BPPV) after closed sinus floor elevation procedure performed with mallet driven osteotomes $^{(30,31) \text {. }}$

the use of screwable osteotomes instead of mallet osteotomes because of their less traumatic action and better hand control, thus helping to avoid the onset of $\mathrm{BPPV}^{(32)}$ suggested. To the best of our knowledge, osseodensification (OD) technique is an alternative treatment modality for crestal sinus floor elevation which also could potentially decrease overall treatment time of posterior maxilla to a great value while introducing less trauma and postoperative discomforts.

Conclusion: current study concluded that:

1- Osseodensification (OD) technique is an alternative treatment modality for crestal sinus floor elevation.

2- Osseodensification (OD) technique produces less pain after surgery and has higher acceptance by patients in comparison to osteotome technique.

3- There was no significant difference between osseodensification (OD) and osteotome in sinus floor elevation regarding implant stability and amount of gained graft height apically throughout different time intervals of follow-up.

\section{REFERENCES}

1. Ahmed, A.K., 2019. ASSESSMENT OF DENTAL IMPLANTS PLACED IN POSTERIOR MAXILLARY RIDGE USING DENSAH BURS VERSUS STANDARD DRILLS (RANDOMIZED CLINICAL TRIAL). CU Theses. 
2. Hassan, M.A., 2016. Evaluation of Reamer Mediated Crestal Sinus Floor Elevation with Simultaneous Implant Placement in Periodontally Compromised Subjects With and Without Porous Titanium Granules:(Clinical and Radio graphical Study). EC Dental Science, 3, pp.658-671.

3. Tallarico, M., Xhanari, E. and Meloni, P.P.S.M., Minimally invasive hydraulic elevation of the Schneiderian membrane and insertion of bone graft material using a novel self-tapping implant system: radiographic and prosthetic aspects.

4. Yamada, Y., Nakamura-Yamada, S., Miki, M., Nakajimaa, Y. and Babaa, S., Trends in clinical trials on bone regeneration in dentistry-towards an innovative development in dental implant treatment.

5. Kim, Y.K., Lee, J.Y., Park, J.W., Kim, S.G. and Oh, J.S., 2017. Sinus membrane elevation by the crestal approach using a novel drilling system. Implant Dentistry, 26(3), pp.351-356.

6. Cruz, R.S., Lemos, C.A.D.A., Batista, V.E.D.S., GOMES, J.M.D.L., PELLIZZER, E.P. and VERRI, F.R., 2018. Short implants versus longer implants with maxillary sinus lift. A systematic review and metaanalysis. Brazilian oral research, 32.

7. Arafat, S.W. and A Elbaz, M., 2019. Clinical and radiographic evaluation of osseodensification versus osteotome for sinus floor elevation in partially atrophic maxilla: A prospective long term study. Egyptian Dental Journal, 65(1-January (Oral Surgery)), pp.189-195.

8. Silva, L.D., De Lima, V.N., Faverani, L.P., De Mendonca, M.R., Okamoto, R. and Pellizzer, E.P., 2016. Maxillary sinus lift surgery—with or without graft material? A systematic review. International journal of oral and maxillofacial surgery, 45(12), pp.1570-1576. 
9. Schiegnitz, E., Kämmerer, P.W., Sagheb, K., Wendt, A.J., Pabst, A., AlNawas, B. and Klein, M.O., 2017. Impact of maxillary sinus augmentation on oral health-related quality of life. International journal of implant dentistry, 3(1), pp.1-8.

10. Macedo, R.L.L., Ponzoni, D., Vedovatto, E., de Carvalho, F.A. and de Carvalho, P.S.P., 2020. Calvarial graft resorption index in reconstruction of the maxillary sinus of atrophic maxilla. A prospective clinical study. British Journal of Oral and Maxillofacial Surgery.

11. Yan, M., Liu, R., Bai, S., Wang, M., Xia, H. and Chen, J., 2018. Transalveolar sinus floor lift without bone grafting in atrophic maxilla: A meta-analysis. Scientific reports, 8(1), pp.1-9.

12. Duan, D.H., Fu, J.H., Qi, W., Du, Y., Pan, J. and Wang, H.L., 2017. Graftfree maxillary sinus floor elevation: A systematic review and metaanalysis. Journal of periodontology, 88(6), pp.550-564.

13. Aldahlawi, S., Demeter, A. and Irinakis, T., 2018. The effect of implant placement torque on crestal bone remodeling after 1 year of loading. Clinical, cosmetic and investigational dentistry, 10, p.203.

14. Sánchez-Siles, M., Ilha, J.B., Ruizc, J.A.F., Alonsod, F.C. and Alonso, F.C., 2019. Evaluation of primary stability and early healing of 2 implant macro designs placed in the posterior maxilla: a split-mouth prospective randomized controlled clinical study. J Oral Rehabil, 5, pp.8-15.

15. Bajaj, G., Bathiya, A., Gade, J., Mahale, Y., Ulemale, M. and Atulkar, M., 2017. Primary versus Secondary Implant Stability in Immediate and Early Loaded Implants. Int J Oral Health Med Res, 3(5), pp.49-54.

16. Zanetti, E.M., Pascoletti, G., Calì, M., Bignardi, C. and Franceschini, G., 2018. Clinical assessment of dental implant stability during follow-up: what is actually measured, and perspectives. Biosensors, 8(3), p.68. 
17. Zanetti, E.M., Pascoletti, G., Calì, M., Bignardi, C. and Franceschini, G., 2018. Clinical assessment of dental implant stability during follow-up: what is actually measured, and perspectives. Biosensors, 8(3), p.68.

18. Bafijari, Denis, Alberto Benedetti, Aleksandar Stamatoski, Florent Baftijari, Zoran Susak, and Darko Veljanovski. "Influence of Resonance Frequency Analysis (RFA) Measurements for Successful Osseointegration of Dental Implants During the Healing Period and Its Impact on Implant Assessed by Osstell Mentor Device." Open access Macedonian journal of medical sciences 7, no. 23 (2019): 4110.

19. Shihab, O.I., 2017. Intentional penetration of dental implants into the maxillary sinus: a retrospective study. Zanco Journal of Medical Sciences (Zanco J Med Sci), 21(1), pp.1536-1539.

20- Karacayli, U., Dikicier, E. and Dikicier, S., 2015. Dental implant placement in inadequate posterior maxilla. Current Concepts in Dental Implantology. Rijeka, Croatia: InTech, pp.105-125.

21- Marley-320 (2019). Crestal Sinus Elevation For Implant Placement. [online] Oral Health Group. Available at: https://www.oralhealthgroup.com/features/crestal-sinus-elevation-forimplant-placement/ [Accessed 16 May 2021].

22- $\quad$ Mittal, Y., Jindal, G. and Garg, S., 2016. Bone manipulation procedures in dental implants. Indian Journal of Dentistry, 7(2), p.86.

23- Juodzbalys, G., 2019. Regenerative bone potential after sinus floor elevation using various bone graft materials: a systematic review. Quintessence Int, 50, pp.548-558.

24- Thoma, D.S., Haas, R., Tutak, M., Garcia, A., Schincaglia, G.P. and Hämmerle, C.H., 2015. Randomized controlled multicentre study comparing short dental implants $(6 \mathrm{~mm})$ versus longer dental implants (11-15 mm) in combination with sinus floor elevation procedures. Part 1: demographics and patient-reported outcomes at 1 year of loading. Journal of clinical periodontology, 42(1), pp.72-80. 
25- Mangano, C., Sinjari, B., Shibli, J.A., Mangano, F., Hamisch, S., Piattelli, A., Perrotti, V. and Iezzi, G., 2015. A human clinical, histological, histomorphometrical, and radiographical study on biphasic HA-Beta-TCP 30/70 in maxillary sinus augmentation. Clinical Implant Dentistry and Related Research, 17(3), pp.610-618.

26- Turkyilmaz, I., Tumer, C., Ozbek, E.N. and Tözüm, T.F., 2007.

Relations between the bone density values from computerized tomography, and implant stability parameters: a clinical study of 230 regular platform implants. Journal of clinical periodontology, 34(8), pp.716-722.

27- Alfadda, S.A., 2014. Early and immediate loading protocols for overdentures in completely edentulous maxillas: a comprehensive review of clinical trials. J Contemp Dent Pract, 15(6), pp.797-805.

28- Kim, J.M., Kim, S.J., Han, I., Shin, S.W. and Ryu, J.J., 2009. A comparison of the implant stability among various implant systems: clinical study. The journal of advanced prosthodontics, 1(1), p.31.

29--Bernstein, D.N., Kelly, M., Houck, J.R., Ketz, J.P., Flemister, A.S., DiGiovanni, B.F., Baumhauer, J.F. and Oh, I., 2019. PROMIS pain interference is superior vs numeric pain rating scale for pain assessment in foot and ankle patients. Foot \& ankle international, 40(2), pp.139144.

30- Al-Almaie, S., Kavarodi, A.M. and Al Faidhi, A., 2013. Maxillary sinus functions and complications with lateral window and osteotome sinus floor elevation procedures followed by dental implants placement: 
A retrospective study in 60 patients. The journal of contemporary dental practice, 14(3), p.405.

31- Vernamonte, S., Mauro, V. and Messina, A.M., 2011. An unusual complication of osteotome sinus floor elevation: benign paroxysmal positional vertigo. International journal of oral and maxillofacial surgery, 40(2), pp.216-218.

32- Sammartino, G., Mariniello, M. and Scaravilli, M.S., 2011. Benign paroxysmal positional vertigo following closed sinus floor elevation procedure: mallet osteotomes vs. screwable osteotomes. A triple blind randomized controlled trial. Clinical oral implants research, 2011,22(6), pp.669-672. 